ARTICLE

\title{
Semiconductive microporous hydrogen-bonded organophosphonic acid frameworks
}

Patrik Tholen', Craig A. Peeples ${ }^{2}$, Raoul Schaper ${ }^{3}$, Ceyda Bayraktar ${ }^{4}$, Turan Selman Erkal ${ }^{5}$, Mehmet Menaf Ayhan (10 ${ }^{4}$, Bünyemin Çoșut ${ }^{4}$, Jens Beckmann (D) ${ }^{6}$, A. Ozgur Yazaydin (D) ${ }^{5}$, Michael Wark ${ }^{3}$, Gabriel Hanna ${ }^{2}$ Yunus Zorlu (iD ${ }^{4 凶} \&$ Gündoğ Yücesan (1) ${ }^{1 凶}$

Herein, we report a semiconductive, proton-conductive, microporous hydrogen-bonded organic framework (HOF) derived from phenylphosphonic acid and 5,10,15,20-tetrakis[pphenylphosphonic acid] porphyrin (GTUB5). The structure of GTUB5 was characterized using single crystal X-ray diffraction. A narrow band gap of $1.56 \mathrm{eV}$ was extracted from a UVVis spectrum of pure GTUB5 crystals, in excellent agreement with the $1.65 \mathrm{eV}$ band gap obtained from DFT calculations. The same band gap was also measured for GTUB5 in DMSO. The proton conductivity of GTUB5 was measured to be $3.00 \times 10^{-6} \mathrm{~S} \mathrm{~cm}^{-1}$ at $75^{\circ} \mathrm{C}$ and $75 \%$ relative humidity. The surface area was estimated to be $422 \mathrm{~m}^{2} \mathrm{~g}^{-1}$ from grand canonical Monte Carlo simulations. XRD showed that GTUB5 is thermally stable under relative humidities of up to $90 \%$ at $90^{\circ} \mathrm{C}$. These findings pave the way for a new family of organic, microporous, and semiconducting materials with high surface areas and high thermal stabilities.

\footnotetext{
${ }^{1}$ Technische Universität Berlin, Gustav-Meyer-Allee 25, 13355 Berlin, Germany. ${ }^{2}$ University of Alberta, 116 St. and 85 Ave., Edmonton, AB T6G 2R3, Canada.

${ }^{3}$ Carl von Ossietzky Universität Oldenburg, Carl-von-Ossietzky Str. 9-11, 26129 Oldenburg, Germany. ${ }^{4}$ Gebze Technical University, Kimya Bölümü, 41400 Gebze-Kocaeli, Turkey. ${ }^{5}$ University College London, Torrington Place, London WC1E 7JE, UK. ${ }^{6}$ Universität Bremen, Leobener Str. 7, 28359 Bremen, Germany.

凶email: yzorlu@gtu.edu.tr; yuecesan@tu-berlin.de
} 
M etal-organic frameworks (MOFs) emerged as revolutionary microporous materials at the beginning of the 21 st century ${ }^{1-3}$. Owing to their well-ordered pores, which are surrounded by inorganic and organic components, MOFs have been used in a wide range of applications, such as gas storage/separation ${ }^{4-7}$, catalysis ${ }^{8-13}$, magnetism ${ }^{14-16}$, electric conductivity ${ }^{17-19}$, proton conductivity ${ }^{20-22}$, and drug delivery ${ }^{23-25}$. In parallel to MOF research, another closely related family of supramolecular architectures known as hydrogen-bonded organic frameworks (HOFs) has attracted immense interest in recent years ${ }^{26-28}$. In $\mathrm{HOFs}$, the linker connectivity is achieved via hydrogen-bonded networks rather than inorganic building units (IBUs) $^{29-33}$. Hydrogen bonds provide simpler connectivity options compared to the complex molecular, one-dimensional, twodimensional, and three-dimensional IBUs of MOFs ${ }^{34}$. Therefore, the design and synthesis of stable hydrogen-bonded supramolecular networks can be more easily achieved compared to that of MOFs. HOFs are also more convenient to recycle and are free of heavy metal ions, providing environmentally friendly solutions. The recent interest in HOFs has resulted in several review articles $^{35-37}$ summarizing their applications in $\mathrm{CO}_{2}$ capture ${ }^{38-40}$ and proton conductivity ${ }^{41,42}$. However, to date, no semiconducting HOFs have been reported in the literature. Thermally stable and permanently microporous semiconducting HOFs could revolutionize the design of supercapacitors and electrodes due to their simpler chemistry compared to MOFs. In this communication, we present the first example of a HOF (known as GTUB5, where TUB stands for Technische Universität Berlin and G for Gebze), synthesized using phosphonic acid functional groups $\left(\mathrm{R}-\mathrm{PO}_{3} \mathrm{H}_{2}\right)$, which exhibits a low band gap, proton conductivity, and high thermal stability.

The phosphonic acid functional group has two protons and one oxygen from the $\mathrm{P}=\mathrm{O}$ bond, which allow it to form multiple hydrogen bonds with other phosphonic acid groups and thereby stabilize the resulting HOF. Interestingly, the unique structure and multiple metal-binding modes of the phosphonic acid functional group have led to some of the most thermally ${ }^{34,43-46}$ and chemically stable ${ }^{34,47-49}$ MOFs in the literature. The phosphonic acid functional group contains two deprotonation modes with $\mathrm{p} K_{\mathrm{a}}$ values of 1.7 and 7.4, respectively ${ }^{31}$. Therefore, in order to synthesize our phosphonate HOF, we adopted a crystallization method at pHs between 1.7 and 7.4 with mixed phosphonic acid linkers of phenylphosphonic acid (PPA) and 5,10,15,20-tetrakis [ $p$-phenylphosphonic acid] porphyrin $\left(\mathrm{H}_{8}-\mathrm{TPPA}\right)$ to ensure that at least one of the phosphonic acid moieties is not fully deprotonated. $\mathrm{H}_{8}$-TPPA exhibits a planar tetratopic geometry with a $90^{\circ}$ angle between the phenylphosphonate tethers ${ }^{49,50}$. Given these starting conditions and materials, it is expected that a mixed linker strategy involving $\mathrm{H}_{8}$-TPPA and PPA could produce twodimensional HOFs with hexagonal void channels.

\section{Results}

Design and structural characterization. The $\mathrm{H}_{8}$-TPPA linker was synthesized according to our previously reported method involving a Pd-catalyzed Arbuzov reaction ${ }^{50}$, in order to avoid the porphyrin core being occupied by $\mathrm{Ni}(\mathrm{II})$ after a $\mathrm{Ni}$-catalyzed Arbuzov reaction ${ }^{49,50}$. The synthesized metal-free $\mathrm{H}_{8}$ TPPA linker eliminated the possibility of metal-ligand interactions that could have triggered the formation of MOFs. GTUB5 was synthesized following a conventional MOF crystallization method in scintillation vials in $\mathrm{DMF} / \mathrm{EtOH}$ at a $\mathrm{pH}$ between 1.7 and 7.44 to ensure the presence of protonated phosphonic acid functional groups ${ }^{32}$. The synthesis of GTUB5 is quite facile, giving dark purple 1-2 $\mathrm{mm}$ long needle-shaped crystals in high yield (see "Methods" section for synthesis details). The dark purple color of GTUB5 is an indication of its semiconductive nature. The structure of GTUB5 was characterized using single crystal X-ray diffraction. As seen in Fig. 1a, b, GTUB5 is composed of two-dimensional sheets of hydrogen-bonded $\mathrm{H}_{8}$-TPPA and PPA moieties. The structure contains two different hydrogen-bonding patterns, which are observed between different $\mathrm{H}_{8}$-TPPA units and between $\mathrm{H}_{8}$-TPPA and PPA (see Fig. 1e). In the first pattern, the $\mathrm{P}=\mathrm{O}$ bond from the $\mathrm{H}_{8}$-TPPA unit is exclusively involved in creating the (almost linear) double hydrogen-bonding pattern between each unit. In the second pattern, the hydrogen bond forms between the second protonated hydroxyl group of the $\mathrm{H}_{8}$ TPPA and the deprotonated $\mathrm{PPA}^{2-}$. The four DMF solvent molecules in the HOF structure act as a Lewis base acquiring the PPAs' protons. The Brunauer-Emmett-Teller (BET) surface area of GTUB5 was estimated to be $422 \mathrm{~m}^{2} \mathrm{~g}^{-1}$ from a simulated $\mathrm{N}_{2}$ adsorption isotherm at $77 \mathrm{~K}$ (see Supplementary Fig. 3) obtained using the grand canonical Monte Carlo method (see "Methods" section for simulation details).

Band gap measurements. The band gap was estimated from a solid-state diffuse reflectance UV-Vis spectrum of the GTUB5 crystals (see Supplementary Fig. 8). As seen in Fig. 1c, the Tauc plot derived from the spectrum yields a narrow band gap of 1.56 $\mathrm{eV}$. The second jump at $2.88 \mathrm{eV}$ corresponds to the Soret band of the porphyrin core at $430 \mathrm{~nm}$. A similar band gap was also obtained from a UV-Vis spectrum of a dissolved sample of GTUB5 in DMSO (see Supplementary Fig. 7), suggesting that the hydrogen-bonded supramolecular structure is not disrupted in a polar aprotic solvent. From a cyclic voltammetry measurement on GTUB5 in DMSO (see Supplementary Fig. 9), the first oxidation and reduction potentials were measured to be 0.42 and $-1.23 \mathrm{~V}$, respectively, yielding a HOMO-LUMO gap of $1.65 \mathrm{eV}$, thereby supporting this hypothesis.

Density functional theory (DFT) calculations. To gain insight into the semiconductive nature of GTUB5, we performed DFT calculations. The details of the calculations, employing hybrid Gaussian plane-wave (GPW) and Slater-type orbital (STO) basis sets, can be found in the "Methods" section. Figure 2 shows a periodic representation of the optimized geometry, which is in close agreement with the experimental crystal structure (see Supplementary Table 3 and Supplementary Figs. 4-7). A single point calculation on the optimized structure yields a band gap of $1.65 \mathrm{eV}$, in very good agreement with the experimental result of $1.56 \mathrm{eV}$. As seen in Fig. 2, the HOMO and LUMO are predominantly localized on some of the porphyrins within the supercell (in which, a single unit cell is delineated by the black rectangle), but not all of them; with the LUMO localized on the same porphyrins as the HOMO. The fact that the HOMO and LUMO are aligned along the axis perpendicular to the plane of the page within the supercell suggests that GTUB5 is only conductive along this direction.

Focusing in on the portions of the structure that have significant HOMO and LUMO density, we see that the HOMO and LUMO are indeed localized on the same porphyrin (see Fig. 3). Moreover, they are mostly confined to a subset of the carbons and nitrogens. The HOMO is composed of $\pi$ orbitals mostly on $s p^{2}$ hybridized carbons and nitrogens, while the LUMO is composed of $\pi^{*}$ orbitals on some of the $s p^{2}$ carbons and nitrogens. As shown in Table 1, 75\% of the HOMO and LUMO orbital contributions are from the carbon and nitrogen $2 p$ orbitals of the porphyrin. Table 1 also shows that a HOMO-LUMO transition would lead to an increase in the carbon $2 p_{x}$ orbital population, a slight decrease in the carbon $2 p_{y}$ population, and a slight increase in the carbon $2 p_{z}$ population; while the nitrogen 
a

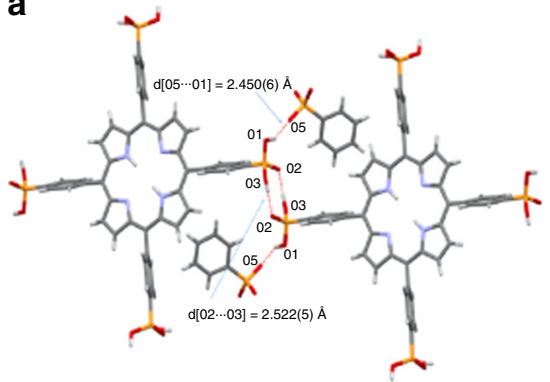

d

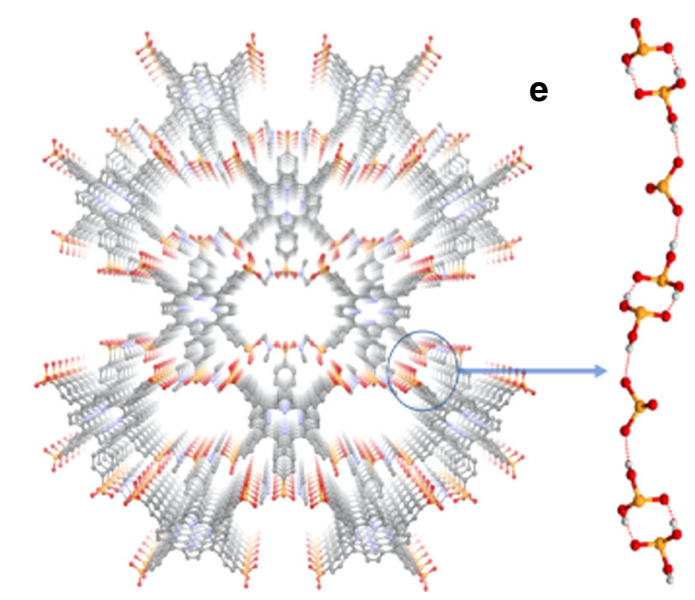

C

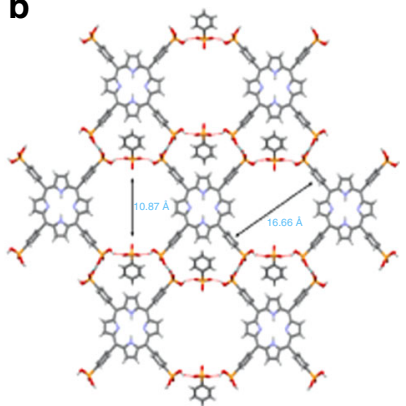

$\operatorname{lic}_{-\infty}^{-\infty}$

f

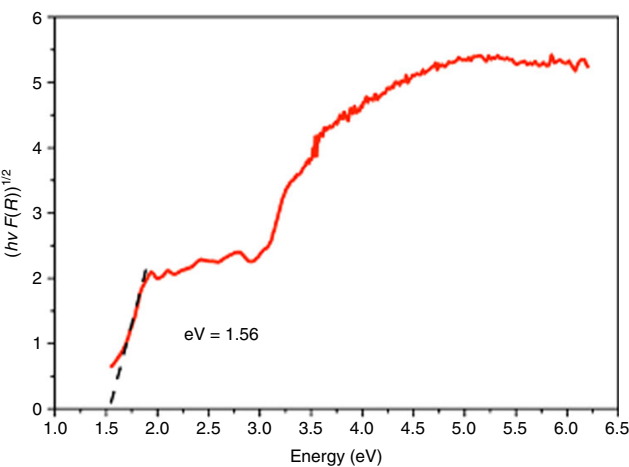

for

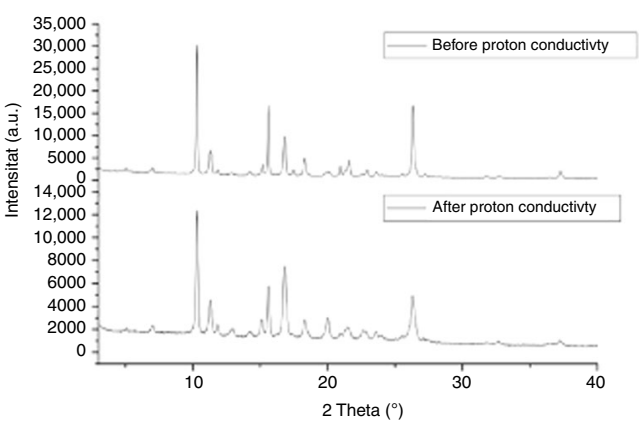

Fig. 1 Structure of GTUB5, experimental band gap, and XRD patterns. a Portion of hydrogen-bonded network of GTUB5. b Depiction of hexagonal void spaces in GTUB5. c Tauc plot from the solid-state UV-Vis spectrum of GTUB5, showing a band gap of $1.56 \mathrm{eV}$. The second jump at $2.88 \mathrm{eV}$ corresponds to the Soret band of the porphyrin core at $430 \mathrm{~nm}$. $\mathbf{d}$ Layer structure of GTUB5. e One-dimensional hydrogen-bonded building unit of GTUB5. $\mathbf{f}$ XRD pattern before and after the proton conductivity measurements.

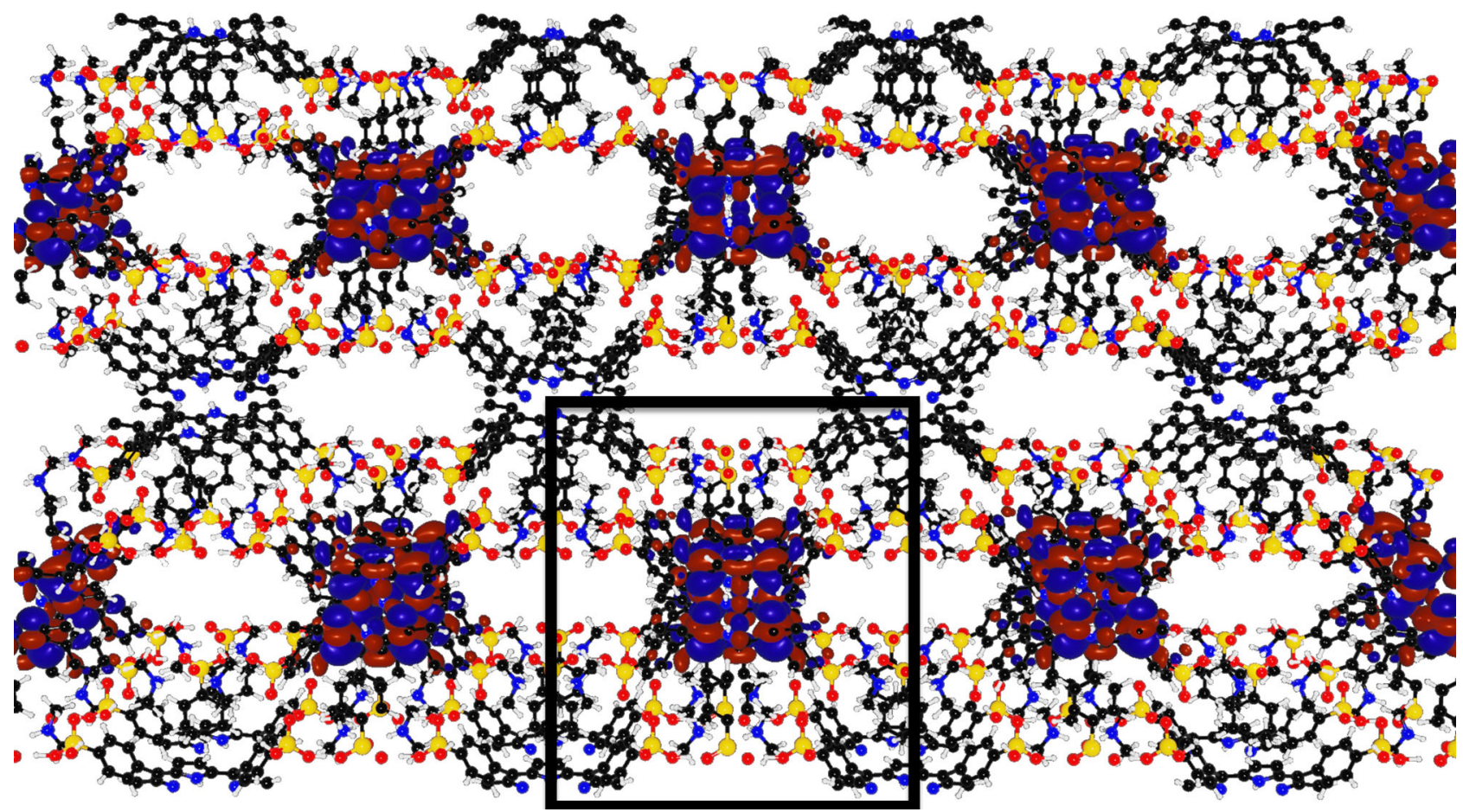

Fig. 2 Periodic representation of GTUB5, with the unit cell delineated by the black box. The HOMO iso-surface corresponding to an electron density of 0.01 electrons per $\AA^{3}$ (negative and positive phases are shown in red and blue, respectively) is also shown ( $\mathrm{O}-$ red; $\mathrm{N}-$ blue; $\mathrm{P}-$ yellow; $\mathrm{C}-$ black; $\mathrm{H}-$ white). 

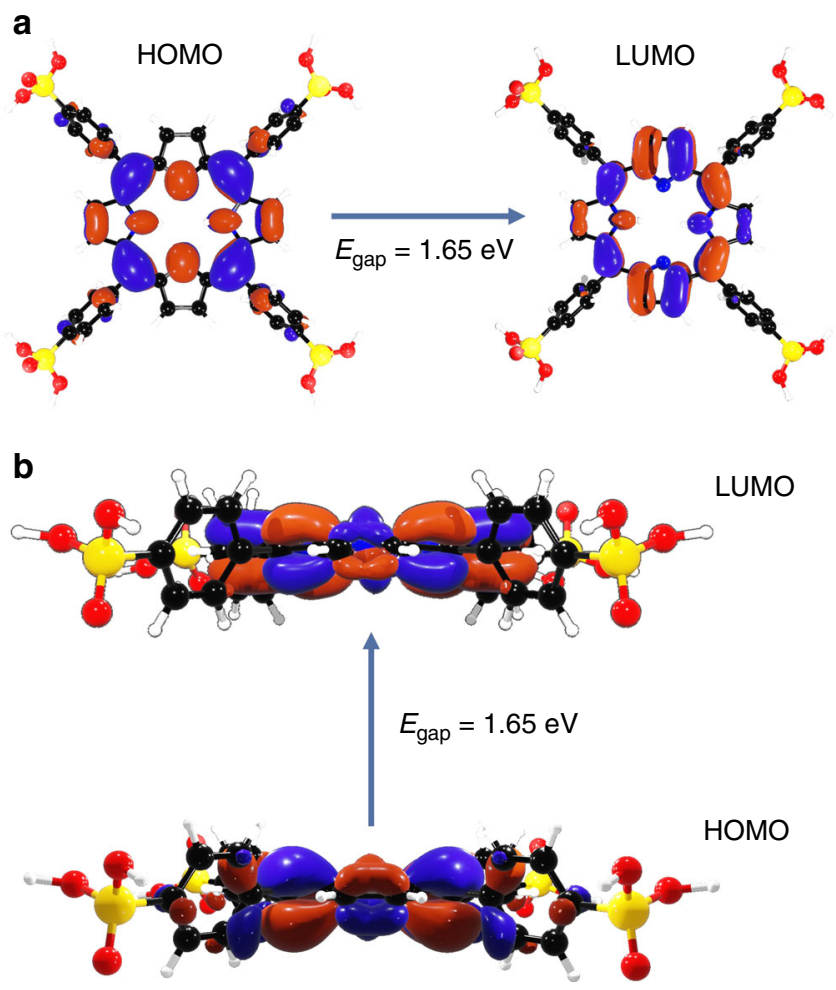

Fig. 3 HOMO and LUMO iso-surfaces, corresponding to an electron density of $\mathbf{0 . 0 1}$ electrons per $\AA^{\mathbf{3}}$. a Top view. b Side view. Red/blue correspond to the negative/positive phases ( $\mathrm{O}-$ red; $\mathrm{N}-$ blue; $\mathrm{P}$-yellow; C-black; H-white)

Table 1 Contributions from the $2 p$ orbitals on the porphyrin carbons and nitrogens to the HOMO and LUMO.

\begin{tabular}{lllll} 
& $\mathbf{2 p}_{\boldsymbol{x}}$ & $\mathbf{2} \boldsymbol{p}_{\mathbf{y}}$ & $\mathbf{2} \boldsymbol{p}_{\mathbf{z}}$ & Sum \\
\hline Carbon & & & & \\
HOMO & 0.366 & 0.042 & 0.134 & 0.541 \\
LUMO & 0.484 & 0.020 & 0.170 & 0.674 \\
Nitrogen & & & & \\
HOMO & 0.163 & $4.70 \times 10^{-7}$ & 0.053 & 0.216 \\
LUMO & 0.048 & $5.22 \times 10^{-4}$ & 0.020 & 0.067 \\
\hline
\end{tabular}

$2 p_{x}$ and $2 p_{z}$ populations both decrease (the $2 p_{y}$ population remains negligible). These results suggest that the semiconductive nature of GTUB5 is predominantly determined by $\pi-\pi^{*}$ transitions involving orbitals localized on some of the porphyrin carbons and nitrogens. Inspection of the projected density of states (pDOS) confirms that the HOMO-LUMO gap is predominantly due to orbitals localized on carbons and nitrogens (see Fig. 4).

Thermogravimetric analysis. The thermal decomposition of GTUB5 was investigated via a thermogravimetric analysis (TGA) of hand-picked crystals up to $900^{\circ} \mathrm{C}$. The TGA curve (see Supplementary Fig. 10) shows an initial 2\% loss between 50 and $100^{\circ} \mathrm{C}$, corresponding to the evaporation of the remaining $\mathrm{MeOH}$ on the crystal surface after the synthesis. The following $12 \%$ step until $250^{\circ} \mathrm{C}$ corresponds to the loss of dimethylammonium cations (calculated to be $12.9 \%$ based on the molecular formula). The remaining organic components of GTUB5 decompose in two steps at ca. 400 and $900^{\circ} \mathrm{C}$. The second large weight loss at ca. $900^{\circ} \mathrm{C}$ suggests the formation of phosphides above $400{ }^{\circ} \mathrm{C}^{52}$.
Proton conductivity. Given the presence of $-\mathrm{PO}_{3} \mathrm{H}_{2}$ groups in its hydrogen-bonded framework, the proton conductivity of GTUB5 was measured. Electrochemical impedance spectroscopy measurements were carried out at $75 \%$ and $90 \%$ relative humidity (\% rh) and temperatures in the range of $25-75^{\circ} \mathrm{C}$ (see Supplementary Information and ref. ${ }^{52}$ for setup details). At $75 \%$ rh, we see that the proton conductivity of GTUB5 increases from $8.29 \times$ $10^{-7}$ to $3.00 \times 10^{-6} \mathrm{~S} \mathrm{~cm}^{-1}$ as the temperature is increased from 25 to $75^{\circ} \mathrm{C}$, while at $90 \%$ rh the conductivities are higher but the increase is more moderate (see Table 2 for full data set). The activation energies, i.e., sum of the migration and defect formation energies, were extracted from the slopes of the Arrhenius plots (see Supplementary Fig. 15) to be $E_{\mathrm{A}}=0.26 \mathrm{eV}$ and $E_{\mathrm{A}}=$ $0.14 \mathrm{eV}$ at 75 and $90^{\circ} \mathrm{C}$, respectively. These low activation energies suggest that a Grotthuss mechanism with high proton mobility (and therefore low migration energy) and $\mathrm{H}$-bridges to water molecules is the predominant mechanism for proton conduction through the framework. As seen in Fig. 1f, the XRD pattern of the sample recorded before and after the proton conductivity experiments slightly changes, indicating that the structure was slightly affected by the humidified atmosphere and the applied temperatures up to $75^{\circ} \mathrm{C}$ during the measurements.

Due to the large numbers of phosphonate groups and hydrogen bonds in GTUB5, one might expect higher proton conductivities. However, in addition to the numbers of phosphonate groups and hydrogen bonds, one must also (and more importantly) consider how rigidly the phosphonate groups are connected to the framework, i.e., how much they change their positions (through rotations and vibrations), and, in turn, how strongly they interact with the water molecules. In a previous study, we found comparable proton conductivities (viz., $1.35 \times 10$ ${ }^{-6} \mathrm{~S} \mathrm{~cm}^{-1}$ at $75 \%$ rh and $80^{\circ} \mathrm{C}$ and $5.62 \times 10^{-6} \mathrm{~S} \mathrm{~cm}^{-1}$ at $90 \% \mathrm{rh}$ and $80^{\circ} \mathrm{C}$ ) for porous porphyrin-based metal tetraphosphonates of the CAU-29 type whose phosphonates sit similarly close to the network ${ }^{53}$. If, however, proton-conducting groups are linked to the framework via flexible vibrating and rotating alkyl chains, like in $\mathrm{SO}_{3} \mathrm{H}$-propylsilane modified Si-MCM-41, proton conductivities higher than $10^{-3} \mathrm{~S} \mathrm{~cm}^{-1}$ at $98 \%$ rh and $80^{\circ} \mathrm{C}$ could be achieved $^{54}$. As quantum chemical calculations in that study confirmed, the proton conductivity is not only strongly dependent on the density of the proton-conducting groups per $\mathrm{nm}^{2}$, but also on the length of the alkyl chain connecting the proton-conducting group with the rigid framework. In the cases of GTUB5 and CAU-29, no such flexible alkyl chains are present.

\section{Discussion}

Herein, we presented GTUB5, a two-dimensional, microporous phosphonic acid HOF (with a calculated surface area of $422 \mathrm{~m}^{2} \mathrm{~g}^{-1}$ ). Given its low band gap (as confirmed by solid-state/solution measurements and DFT calculations), GTUB5 paves the way for the creation of new semiconductive microporous organic compounds. The use of hydrogen bonds in constructing a framework comes with the advantages of simpler connectivity options and no toxic metal ions (which could possibly lead to environmentally friendly solutions for capacitors and batteries). The hydrogen-bonded framework also renders GTUB5 proton-conductive (with proton conductivities on the order of $10^{-6} \mathrm{~S} \mathrm{~cm}^{-1}$ ). Owing to its structure, the phosphonic acid group can give rise to structurally diverse frameworks, which could increase the number of potential HOF applications. GTUB5 has the same band gap of $1.65 \mathrm{eV}$ in DMSO, suggesting that GTUB5 retains its hydrogen-bonded network in polar aprotic solvents. Therefore, phosphonate HOFs have the potential to revolutionize the semiconductive materials industry with applications in printed electronics, optoelectronics, photovoltaics, and electrodes in supercapacitors. Currently, we are focusing on different linker geometries 


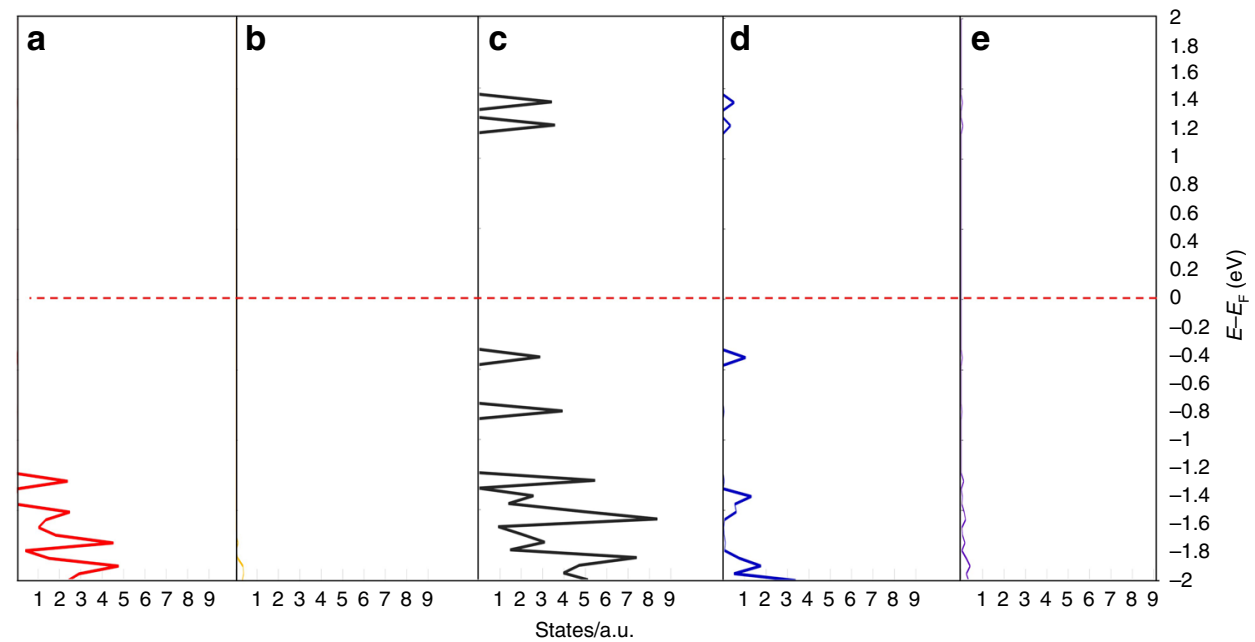

Fig. 4 Atom-specific projected density of states (pDOS) for GTUB5, generated using ADF-BAND51. Projected density of states (pDOS) for a O, b P, c C, d $\mathrm{N}$, and $\mathbf{e} \mathrm{H}$ in GTUB5, generated using ADF-BAND ${ }^{51}$.

\section{Table 2 Proton conductivities and activation energies $\left(E_{A}\right)$ of GTUB5 at different relative humidities.}

\begin{tabular}{|llll|} 
Relative humidity [\%rh] & & $\mathbf{7 5}$ & $\mathbf{9 0}$ \\
\hline Conductivity $\left[\mathrm{S} \mathrm{cm}^{-1}\right]$ & $25^{\circ} \mathrm{C}$ & $8.29 \times 10^{-7}$ & $3.55 \times 10^{-6}$ \\
& $50^{\circ} \mathrm{C}$ & $1.67 \times 10^{-6}$ & $3.26 \times 10^{-6}$ \\
& $75^{\circ} \mathrm{C}$ & $3.00 \times 10^{-6}$ & $4.20 \times 10^{-6}$ \\
$E_{\mathrm{A}}[\mathrm{eV}]$ & & 0.26 & 0.14 \\
\hline
\end{tabular}

and $\mathrm{pH}$ modulations to further optimize the pore sizes and conductive behavior of phosphonate HOFs.

\section{Methods}

Synthesis. All the reagents and solvents employed were commercially available and used as received without further purification. The linker $\mathrm{H}_{8}$ TPPA was synthesized according to our previously reported method ${ }^{50}(8.77 \mathrm{mg}, 0.0088 \mathrm{mmol})$ and phenylphosphonic acid (PPA) $(208 \mathrm{mg}, 1.3 \mathrm{mmol})$ in a $1.6 \mathrm{~mL}$ mixture of DMF/EtOH or DMF/MeOH (1.36:0.24, v/v) were added to a $5-\mathrm{mL}$ glass vial. The reaction mixture was ultrasonically dissolved and then heated to $80^{\circ} \mathrm{C}$ in an oven for $48 \mathrm{~h}$. After cooling down to room temperature, dark purple block crystals of GTUB5 formed, which were then isolated by filtration, washed with DMF and acetone, and finally air-dried. The yield of GTUB5 was $5 \mathrm{mg}$.

Single crystal structure solution. Suitable single crystals of GTUB5 with appropriate dimensions $(0.43 \mathrm{~mm} \times 0.14 \mathrm{~mm} \times 0.12 \mathrm{~mm})$ were carefully chosen from the glass vial using a polarizing microscope, coated with perfluoropolyether oil in order to eliminate the possibility of decomposition, and finally mounted to a thin glass fiber. Intensity data collection was performed with a Bruker APEX II QUAZAR three-circle diffractometer equipped with the I $\mu$ S Incoatec Microfocus Source with Mo-Ka radiation $(\lambda=0.710723 \AA)$ at room temperature $(296 \mathrm{~K})$. Indexing was performed using APEX2 ${ }^{55}$. Data integration and reduction were carried out with SAINT ${ }^{56}$. Absorption correction was performed by multi-scan method implemented in SADABS ${ }^{57}$. The structure was solved using SHELXT ${ }^{58}$ and then refined by full-matrix least-squares refinements on $\mathrm{F}^{2}$ using SHELXL ${ }^{59}$ in the Olex2 Software Package ${ }^{60}$. The positions of all $\mathrm{H}$-atoms bonded to the carbon, nitrogen, and oxygen atoms were geometrically optimized with the following HFIX instructions in SHELXL: HFIX 23 for the $-\mathrm{CH}_{2}$ - moieties, HFIX 137 for the $-\mathrm{CH}_{3}$, HFIX 43 for the $\mathrm{CH}$ and $\mathrm{NH}$ groups of the aromatic rings and porphyrin cores, and HFIX 147 for the $-\mathrm{P}-\mathrm{OH}$ groups (H1a) of the phosphonic acid moieties. Another O-bound $\mathrm{H}$ atom (H3) was located from the difference Fourier-map. Finally, their displacement parameters were set to isotropic thermal displacements parameters $\left[U_{\text {iso }}(\mathrm{H})=1.2 U_{\text {eq }}\right.$ for $\mathrm{CH}, \mathrm{NH}$, and $\mathrm{CH}_{2}$ groups and $U_{\mathrm{iso}}(\mathrm{H})=1.5 U_{\mathrm{eq}}$ for $\mathrm{OH}$ and $\mathrm{CH}_{3}$ groups]. In the chemical formula $\left[\left(\mathrm{H}_{8}\right.\right.$-TPPA $\left.)(\mathrm{PPA})_{2}(\mathrm{DMA})_{4}\right]$ of GTUB5, the $\mathrm{H}_{8}$-TPPA building block is not deprotonated, while the protons of the phenylphosphonic acid (PPA) groups are acquired by the DMF solvent in the pores to form four dimethylammonium cations (DMA- $\left[\mathrm{NH}_{2}\left(\mathrm{CH}_{3}\right)_{2}\right]^{+}$) to balance the charge. SQUEEZE was used to remove the electron density caused by seriously disordered solvent molecules in GTUB5. Along the $c$-axis, the 3D supramolecular network of GTUB5 produced a one-dimensional distinctive void space with a total potential solvent area occupying $19.2 \%\left(785 \AA^{3}\right)$ of the unit cell volume (4081.7 $\AA^{3}$ ), obtained using the PLATON software package ${ }^{61}$. Analysis of solvent accessible voids in the structure was performed using CALC SOLV in PLATON with a probe radius of $1.20 \AA$ and grid spacing of $0.2 \AA$. Van der Waals (or ion) radii used in the analysis are $1.70 \AA$ for $\mathrm{C}, 1.20 \AA$ for $\mathrm{H}, 1.55 \AA$ for $\mathrm{N}, 1.52 \AA$ for $\mathrm{O}$, and $1.80 \AA$ for $\mathrm{P}$. Also, in this crystal structure, the rotationally disordered phosphonate part $\left(-\mathrm{PO}_{3}\right)$ in PPA was refined as 0.77:0.23. Crystallographic data and refinement details of the data collection for GTUB5 are given in Supplementary Table 4. Crystal structure validations and geometrical calculations were performed using PLATON ${ }^{61}$. The Mercury software package ${ }^{62}$ was used for visualization of the cif files.

Grand canonical Monte Carlo (GCMC) surface area calculations. The surface area of GTUB5 was calculated by force-field based atomistic simulations, which were performed with the RASPA molecular simulation package ${ }^{63}$. For these simulations, the GTUB5 unit cell was replicated by $1 \times 2 \times 4$ times in the $x, y$, and $z$ directions, respectively, and the replicated framework atoms were fixed in their crystallographically determined positions. Lennard-Jones (LJ) and Coulomb potentials were employed to determine the non-bonded interaction energies between atoms:

$$
V_{i j}=4 \varepsilon_{i j}\left[\left(\frac{\sigma_{i j}}{r_{i j}}\right)^{12}-\left(\frac{\sigma_{i j}}{r_{i j}}\right)^{6}\right]+\frac{q_{i} q_{j}}{4 \varepsilon_{0} r_{i j}}
$$

where $r_{i j}$ is the distance between atoms $i$ and $j, \varepsilon_{i j}$ and $\sigma_{i j}$ are the LJ well depth and diameter, respectively, $q_{i}$ is the partial charge of atom $i$, and $\varepsilon_{0}$ is the dielectric constant. The LJ parameters between different types of sites were calculated using the Lorentz-Berthelot mixing rules, and the Ewald summation method was employed to compute the electrostatic interactions. The LJ interactions were shifted to be 0 at a cutoff distance of $12.0 \AA$. For the real part of the Ewald summation, the cutoff was also set to $12.0 \AA$.

A $\mathrm{N}_{2}$ adsorption isotherm for GTUB5 was computed by performing GCMC simulations at $77 \mathrm{~K}$ and up to 0.4 bar. In the GC ensemble, the chemical potential, volume, and temperature of the system are fixed; however, the number of molecules fluctuates. For all GCMC simulations, a 100,000 cycle initialization and a 100,000 cycle production run were performed. Each cycle is $N$ steps, where $N$ is equal to the number of molecules in the system. Random insertions, deletions, translations, rotations, and reinsertions of the $\mathrm{N}_{2}$ molecules were sampled with equal probability. The TraPPE force field was used to model the $\mathrm{N}_{2}$ molecules ${ }^{64}$, which was originally fit to reproduce the vapor-liquid coexistence curve of $\mathrm{N}_{2}$. In this force field, the $\mathrm{N}_{2}$ molecule is rigid with the $\mathrm{N}-\mathrm{N}$ bond length fixed at its experimental value of $1.10 \AA$. This model reproduces the experimental gas-phase quadrupole moment of the $\mathrm{N}_{2}$ molecule by placing partial charges on nitrogen atoms and on a point located at the center of mass (COM) of the molecule. Supplementary Table 2 shows the LJ parameters and partial charges for the $\mathrm{N}_{2}$ molecule. In GCMC simulations, one computes the absolute adsorption $\left(N_{\text {total }}\right)$; whereas, in adsorption experiments, the excess adsorption $\left(N_{\text {excess }}\right)$ is measured. Therefore, the simulated excess adsorption of $\mathrm{N}_{2}$ was calculated using the following expression:

$$
N_{\text {total }}=N_{\text {excess }}+\rho_{\text {gas }} V_{\mathrm{p}}
$$

where $\rho_{\text {gas }}$ is the bulk density of the gas at simulation conditions which were calculated using the Peng-Robinson equation of state and $V_{\mathrm{p}}$ is the accessible pore volume. The BET surface area of GTUB5 was obtained from the simulated $\mathrm{N}_{2}$ 
adsorption isotherm (see Supplementary Fig. 3) and estimated to be $422 \mathrm{~m}^{2} \mathrm{~g}^{-1}$. When applying the BET theory, we made sure that our analysis satisfied the two consistency criteria as detailed by Walton and Snurr ${ }^{65}$.

Density functional theory (DFT) calculations. The geometry optimization of GTUB5 was performed using DFT and the conjugate gradient method ${ }^{66}$ within the Quickstep-CP2K program ${ }^{67,68}$, starting from the experimental crystal structure and with the lattice vectors set to their experimental values. Since GTUB5 is a bulk material, periodic boundary conditions were applied to a reoriented $1 \times 1 \times 1$ cell $\left(a=25.452 \AA, b=22.863 \AA, c=7.1798 \AA, \alpha=\gamma=90.0^{\circ}, \beta=102.325^{\circ}\right)$. The Perdew-Burke-Ernzerhof (PBE) ${ }^{69}$ generalized gradient approximation (GGA) functional was used in conjunction with the Grimme D3 dispersion correction ${ }^{70}$ and BJ damping ${ }^{71}$. The Gaussian and plane waves method 70,72 was used, with the valence orbitals expanded in terms of molecularly optimized Gaussian basis sets of double- $\zeta$ plus polarization (MOLOPT-DZVP) ${ }^{73}$ quality and the core electrons represented by norm-conserving Goedecker-Teter-Hutter pseudopotentials ${ }^{74,75}$. $\Gamma$-point sampling was used and the plane-wave cutoff in reciprocal space was set to 550 Ry, with a Gaussian mapping of 60 Ry over five multi-grids. The self-consistent field was converged to $10^{-6}$ Ry with the FULL_ALL preconditioner using the orbital transformation method with a HOMO-LUMO gap of $1.67 \mathrm{eV}$. Single point calculations were performed using CP2K to obtain the HOMO-LUMO iso-surface plots (Figs. 2 and 3), orbital populations (Table 1), and the HOMO-LUMO gap. Another single point calculation was performed using the Slater-Type Orbital (STO) software ADF-BAND 2018.104 (pDOS) (Fig. 4), band structure (Supplementary Fig. 8), and the HOMO-LUMO gap. The periodic ADF-BAND calculations were performed using an all-electron double- $\zeta$ plus polarization (DZP) basis set, PBE-D3-BJ, and $\Gamma$-point sampling for the $1 \times 1 \times 1$ unit cell, with a good numerical quality. The HOMO-LUMO gaps obtained from CP2K and ADF-BAND were both $1.65 \mathrm{eV}$ (thus, the HOMO-LUMO iso-surfaces and orbital populations obtained from $\mathrm{CP} 2 \mathrm{~K}$ are expected to be the same as those from ADF-BAND).

\section{Data availability}

All data is available in the main text and the Supplementary Information. The source data for Table 1, Fig. 1c, Supplementary Figs. 2-4, 10, 13, 16, 17, and the CIF file are provided as a Source Data file. The X-ray crystallographic coordinates for the structure reported in this study can be obtained free of charge from the Cambridge Crystallographic Data Centre (CCDC) [under the deposition number CCDC: 1963794 for GTUB5] via www.ccdc.cam.ac.uk/data_request/cif.

Received: 12 March 2020; Accepted: 4 June 2020; Published online: 23 June 2020

\section{References}

1. Li, H., Eddaoudi, M., O'Keeffe, M. \& Yaghi, O. M. Design and synthesis of an exceptionally stable and highly porous metal-organic framework. Nature 402, 276-279 (1999).

2. Schneemann, A. et al. Flexible metal-organic frameworks. Chem. Soc. Rev. 43, 6062-6069 (2014).

3. Férey, G., Mellot-Draznieks, C., Serre, C. \& Millange, F. Crystallized frameworks with giant pores: are there limits to the possible? Acc. Chem. Res. 38, 217-225 (2005)

4. Mason, J. A., Veenstra, M. \& Long, J. R. Evaluating metal-organic frameworks for natural gas storage. Chem. Sci. 5, 32-51 (2014).

5. Miller, S. R. et al. Structural transformations and adsorption of fuel-related gases of a structurally responsive nickel phosphonate metal-organic framework, Ni-STA-12. J. Am. Chem. Soc. 130, 15967-15981 (2008).

6. Liu, Y., O'Keeffe, M., Treacy, M. M. \& Yaghi, O. M. The geometry of periodic knots, polycatenanes and weaving from a chemical perspective: a library for reticular chemistry. Chem. Soc. Rev. 47, 4642-4664 (2018).

7. Furukawa, H., Cordova, K. E., O'Keeffe, M. \& Yaghi, O. M. The chemistry and applications of metal-organic frameworks. Science 341, 1230444 (2013).

8. Dhakshinamoorthy, A., Li, Z. \& Garcia, H. Catalysis and photocatalysis by metal organic frameworks. Chem. Soc. Rev. 47, 8134-8172 (2018).

9. Yang, D. \& Gates, B. C. ACS Catal. 9, 1779 (2019).

10. Liu, M., Wu, J. \& Hou, H. Metal-organic framework (MOF)-based materials as heterogeneous catalysts for C-H bond activation. Chem. Eur. J. 25, 2935-2948 (2019).

11. Zhu, L., Liu, X.-Q., Jiang, H.-L. \& Sun, L.-B. Metal-organic frameworks for heterogeneous basic catalysis. Chem. Rev. 117, 8129-8176 (2017).

12. Kang, Y.-S. et al. Metal-organic frameworks with catalytic centers: from synthesis to catalytic application. Coord. Chem. Rev. 378, 262-280 (2019).
13. Chen, X. et al. Sixteen isostructural phosphonate metal-organic frameworks with controlled lewis acidity and chemical stability for asymmetric catalysis. Nat. Commun. 8, 1-9 (2017).

14. Espallargas, G. M. \& Coronado, E. Magnetic functionalities in MOFs: from the framework to the pore. Chem. Soc. Rev. 47, 533-557 (2018).

15. Kurmoo, M. Magnetic metal-organic frameworks. Chem. Soc. Rev. 38, 1353-1379 (2009)

16. Stock, N. \& Biswas, S. Syntheses of metal-organic frameworks (MOFs): routes to various MOF topologies, morphologies, and composites. Chem. Rev. 112, 933-969 (2012)

17. Ko, M., Mendecki, L. \& Mirica, K. A. Conductive two-dimensional metalorganic frameworks as multifunctional materials. Chem. Commun. 54, 7873-7891 (2018).

18. Siemensmeyer, K. et al. Phosphonate metal-organic frameworks: a novel family of semiconductors. Adv. Mater. https://doi.org/10.1002/adma. 202000474 (2020).

19. Sun, L., Campbell, M. G. \& Dincă, M. Electrically conductive porous metalorganic frameworks. Angew. Chem. Int. Ed. 55, 3566-3579 (2016).

20. Taylor, J. M. et al. Facile proton conduction via ordered water molecules in a phosphonate metal-organic framework. J. Am. Chem. Soc. 132, 14055-14057 (2010).

21. Levenson, D. A. et al. Effects of secondary anions on proton conduction in a flexible cationic phosphonate metal-organic framework. Chem. Mater. 32, 679-687 (2020)

22. Pili, S. et al. Proton conduction in a phosphonate-based metal-organic framework mediated by intrinsic "free diffusion inside a sphere". J. Am. Chem. Soc. 138, 6352-6355 (2016)

23. Cai, W. et al. Metal-organic framework-based stimuli-responsive systems for drug delivery. Adv. Sci. 6, 1-20 (2019).

24. Horcajada, P. et al. Metal-organic frameworks in biomedicine. Chem. Rev. 112, 1232-1268 (2012)

25. Hartlieb, K. J. et al. Encapsulation of Ibuprofen in CD-MOF and related bioavailability studies. Mol. Pharm. 14, 1831-1839 (2017).

26. Huang, Q. et al. An exceptionally flexible hydrogen-bonded organic framework with large-scale void regulation and adaptive guest accommodation abilities. Nat. Commun. 10, 1-8 (2019).

27. Yin, Q., Lü, J., Li, H.-F., Liu, T.-F. \& Cao, R. Robust microporous porhyrinbased hydrogen-bonded organic framework for highly selective separation of C2 hydrocarbons versus methane. Cryst. Growth Des. 19, 4157-4161 (2019).

28. Yoon, T.-U. et al. Efficient separation of $\mathrm{C} 2$ hydrocarbons in a permanently porous hydrogen-bonded organic framework. Chem. Commun. 54, 9360-9363 (2018).

29. Bao, S.-S., Shimizu, G. K. H. \& Zheng, L.-M. Proton conductive metal phosphonate frameworks. Coord. Chem. Rev. 378, 577-594 (2019).

30. Shearan, S. J. I. et al. New directions in metal phosphonate and phosphinate chemistry. Crystals 9, 270 (2019).

31. Sevrain, C. M., Berchel, M., Couthon, H. \& Jaffrès, P.-A. Phosphonic acid: preparation and applications. Beilstein J. Org. Chem. 13, 2186-2213 (2017).

32. Schoedel, A. et al. Structures of metal-organic frameworks with rod secondary building units. Chem. Rev. 119, 12466-12535 (2016).

33. Ramaswamy, P., Wong, N. E. \& Shimizu, G. K. H. MOFs as proton conductorschallenges and opportunities. Chem. Soc. Rev. 43, 5913-5932 (2014).

34. Tholen, P., Zorlu, Y., Beckmann, J. \& Yücesan, G. Steps towards the next generation of stable and functional MOFs. Eur. J. Inorg. Chem. 17, 1542-1554 (2020).

35. Lin, R.-B. et al. Multifunctional porous hydrogen-bonded organic framework materials. Chem. Soc. Rev. 48, 1362-1389 (2019).

36. Lou, J., Wang, J.-W., Zhang, J.-H., Lai, S. \& Zhong, D.-C. Hydrogen-bonded organic frameworks: design, structures and potential applications. CrystEngComm 20, 5884-5898 (2018).

37. Hisaki, I., Xin, C., Takahashi, K. \& Nakamura, T. Designing hydrogen-bonded organic frameworks (HOFs) with permanent porosity. Angew. Chem. Int. Ed. 58, 11160-11170 (2019).

38. Wang, H. et al. A flexible microporous hydrogen-bonded organic framework for gas sorption and separation. J. Am. Chem. Soc. 137, 9963-9970 (2015).

39. Patil, R. S., Banerjee, D., Zhang, C., Thallapally, P. K. \& Atwood, J. L. Selective $\mathrm{CO}_{2}$ adsorption in a supramolecular organic framework. Angew. Chem. Int. Ed. 55, 4523-4526 (2016).

40. Luo, X.-Z. et al. A microporous hydrogen-bonded organic framework: exceptional stability and highly selective adsorption of gas and liquid. J. Am. Chem. Soc. 135, 11684-11687 (2013).

41. Karmakar, A. et al. Hydrogen-bonded organic frameworks (HOFs): a new calss of porous crystalline proton-conducting materials. Angew. Chem. Int. Ed. 55, 10667-10671 (2016).

42. Yang, W. et al. Microporous diaminotriazine-decorated porphyrin-based hydrogen-bonded organic framework: permanent porosity and proton conduction. Cryst. Growth Des. 16, 5831-5835 (2016). 
43. Yücesan, G., Zorlu, Y., Stricker, M. \& Beckmann, J. Metal-organic solids derived from arylphosphonic acids. Coord. Chem. Rev. 369, 105-122 (2018).

44. Gagnon, K. J., Perry, H. P. \& Clearfield, A. Conventional and unconventional metal-organic frameworks based on phosphonate ligands: MOFs and UMOFs. Chem. Rev. 112, 1034-1054 (2012).

45. Hermer, N. \& Stock, N. The new triazine-based porous copper phosphonate [Cu3(PPT)(H2O)3]·10H2O. Dalton Trans. 44, 3720-3723 (2015).

46. Zorlu, Y. et al. A cobalt arylphosphonate MOF-superior stability, sorption and magnetism. Chem. Commun. 55, 3053-3056 (2019).

47. Gao, C., Ai, J., Tian, H., Wub, D. \& Sun, Z. An ultrastable zirconiumphosphonate framework as bifunctional catalyst for highly active $\mathrm{CO}_{2}$ chemical transformation. Chem. Commun. 53, 1293-1296 (2017).

48. Zheng, T. et al. Overcoming the crystallization and designability issues in the ultrastable zirconium phosphonate framework system. Nat. Commun. 8, 1-11 (2017).

49. Rhauderwiek, T. et al. Highly stable and porous porphyrin-based zirconiium and hafnium phosphonates-electron crystallography as an important tool for structure elucidation. Chem. Sci. 9, 5467-5478 (2018).

50. Maares, M. et al. Alkali phosphonate metal-organic frameworks. Chem. Eur. J. 25, 11214-11217 (2019).

51. Philipsen, P. H. T. et al. BAND 2018.104, SCM, Theoretical Chemistry (Vrije Universiteit, Amsterdam, The Netherlands, 2018).

52. Homburg, T. et al. Magnesium doped gallium phosphonates Ga1-x Mgx $[\mathrm{H} 3+\mathrm{x}(\mathrm{O} 3 \mathrm{PCH} 2) 3 \mathrm{~N}](\mathrm{x}=0,0.20)$ and the influence on proton conductivity. Z. Anorg. Allg. Chem. 644, 86-91 (2018).

53. Rhauderwiek, T. et al. Crystalline and permanently porous porphyrin-based metal tetraphonates. Chem. Comm. 54, 389-392 (2018).

54. Marschall, R. et al. Detailed simulation and characterization of highly proton conducting sulfonic acid functionalized mesoporous materials under dry and humidified conditions. J. Phys. Chem. C 113, 19218-19227 (2009).

55. APEX2, version 2014.11-0, Bruker (Bruker AXS Inc., Madison, WI, 2014).

56. SAINT, version 8.34A, Bruker (Bruker AXS Inc., Madison, WI, 2013).

57. SADABS, version 2014/5, Bruker (Bruker AXS Inc., Madison, WI, 2014).

58. Sheldrick, G. M. SHELXT-integrated space-group and crystal-structure determination. Acta Crystallogr. A A71, 3-8 (2015).

59. Sheldrick, G. M. Crystal structure refinement with SHELXL. Acta Crystallogr. C C71, 3-8 (2015).

60. Dolomanov, O. V., Bourhis, L. J., Gildea, R. J., Howard, J. A. K. \& Puschmann, H. J. Appl. Crystallogr. 42, 336-338 (2009).

61. Spek, A. L. Structure validation in chemical crystallography. Acta Crystallogr. D D65, 148-155 (2009).

62. Macrae, C. F. et al. Mercury: visualization and analysis of crystal structures. J. Appl. Crystallogr. 39, 453-457 (2006).

63. Dubbeldam, D., Calero, S., Ellis, D. E. \& Snurr, R. Q. RASPA: molecular simulation software for adsorption and diffusion in flexible nanoporous materials. Mol. Simulat. 42, 81-101 (2016).

64. Potoff, J. J. \& Siepmann, J. I. Vapor-liquid equilibria of mixtures containing alkanes, carbon dioxide, and nitrogen. AIChE J. 47, 1676-1682 (2001).

65. Walton, K. S. \& Snurr, R. Q. Applicability of the BET method for determining surface areas of microporous metal-organic frameworks. J. Am. Chem. Soc. 129, 8552-8556 (2007).

66. Hestenes, M. R. \& Stiefel, E. Methods of conjugate gradients for solving linear systems. J. Res. Natl Bur. Stand. 49, 409-436 (1952).

67. VandeVondele, J. et al. Quickstep: fast and accurate density functional calculations using a mixed Gaussian and plane waves approach. Comput. Phys. Commun. 167, 103-128 (2005)

68. Hutter, J., Iannuzzi, M., Schiffmann, F. \& Vandevondele, J. CP2K: atomistic simulations of condensed matter systems. WIRES Comput. Mol. Sci. 4, 15-25 (2014).

69. Perdew, J., Burke, K. \& Ernzerhof, M. Generalized gradient approximation made simple. Phys. Rev. Lett. 77, 3865-3868 (1996).

70. Grimme, S., Antony, J., Ehrlich, S. \& Krieg, H. A consistent and accurate ab initio parametrization of density functional dispersion correction (DFT-D) for the 94 elements H-Pu. J. Chem. Phys. 132, 154104-1-154104-19 (2010).

71. Grimme, S., Ehrlich, S. \& Goerigk, L. Effect of the damping function in dispersion corrected density functional theory. J. Comput. Chem. 32, 1456-1465 (2011).

72. Lippert, G., Hutter, J. \& Parrinello, M. A hybrid Gaussian and plane wave density functional scheme. Mol. Phys. 92, 477-488 (1997).

73. VandeVondele, J. \& Hutter, J. Gaussian basis sets for accurate calculations on molecular systems in gas and condensed phases. J. Chem. Phys. 127, 1141051-114105-9 (2007)

74. Goedecker, S. \& Teter, M. Separable dual-space Gaussian pseudopotentials. Phys. Rev. B 54, 1703-1710 (1996).
75. Hartwigsen, C., Goedecker, S. \& Hutter, J. Relativistic separable dual-space gaussian pseudopotentials from $\mathrm{H}$ to Rn. Phys. Rev. B 58, 3641-3662 (1998).

76. Philipsen, P. H. T. et al. BAND 2018.104, SCM, Theoretical Chemistry (Vrije Universiteit, Amsterdam, The Netherlands, 2018).

77. te Velde, G. et al. Chemistry with ADF. J. Comput. Chem. 22, 931-967 (2001)

\section{Acknowledgements}

The authors thank Dr. Pradip Pachfule from TU-Berlin for his help in measuring the solid state UV-Vis spectrum of GTUB5. Gündoğ Yücesan would like to thank the DFG for funding his work with grant number DFG YU 267/2-1 and DAAD for supporting Prof. Dr. Bünyemin Çoşut's visit to his lab at TU-Berlin. We acknowledge support by the German Research Foundation and the Open Access Publication Fund of TU Berlin. Gabriel Hanna acknowledges support from the Natural Sciences and Engineering Research Council of Canada (NSERC). The DFT calculations were enabled by support provided by WestGrid (www.westgrid.ca) and Compute Canada (www.computecanada.ca).

\section{Author contributions}

G.Y. created the hypothesis, supervised the project, wrote the manuscript and co-wrote/ revised the non-computational parts of the manuscript. P.T. resynthesized the compound, determined the experimental band gap, contributed to the crystal structure refinement, prepared the crystallographic figures, organized the proton conductivity work, and contributed to the text. C.A.P. performed the DFT calculations, generated the corresponding figures/tables, and wrote the initial draft of the corresponding parts of the manuscript. R.S. performed the proton conductivity and powder XRD experiments, and contributed to the text. T.S.E. performed the Monte Carlo simulations. C.B. and M.A. synthesized the linker. B.C. generated the Tauc Plot figures, performed, cyclic voltammetry and obtained UV-Vis in DMSO and contributed to the text in corresponding section. A.O.Y. supervised the Monte Carlo simulations and wrote the corresponding text. J.B. critically read the text and contributed to the manuscript. M.W. supervised the proton conductivity work and wrote the corresponding section. G.H. supervised the DFT calculations, co-wrote the corresponding parts of the manuscript, performed extensive critical revisions of the entire manuscript, and edited the entire manuscript. Y.Z. optimized the crystallization, supervised the crystal refinement, supervised the synthesis of the linker, and generated the text/tables corresponding to the crystallographic refinement and synthesis.

\section{Competing interests}

Gündoğ Yücesan has a patent pending for some of the presented results. The other authors declare no competing interests.

\section{Additional information}

Supplementary information is available for this paper at https://doi.org/10.1038/s41467 020-16977-0.

Correspondence and requests for materials should be addressed to Y.Z. or G.Y.

Peer review information Nature Communications thanks Jon Zubieta and the other, anonymous, reviewer(s) for their contribution to the peer review of this work.

Reprints and permission information is available at http://www.nature.com/reprints

Publisher's note Springer Nature remains neutral with regard to jurisdictional claims in published maps and institutional affiliations.

Open Access This article is licensed under a Creative Commons Attribution 4.0 International License, which permits use, sharing, adaptation, distribution and reproduction in any medium or format, as long as you give appropriate credit to the original author(s) and the source, provide a link to the Creative Commons license, and indicate if changes were made. The images or other third party material in this article are included in the article's Creative Commons license, unless indicated otherwise in a credit line to the material. If material is not included in the article's Creative Commons license and your intended use is not permitted by statutory regulation or exceeds the permitted use, you will need to obtain permission directly from the copyright holder. To view a copy of this license, visit http://creativecommons.org/ licenses/by/4.0/.

(C) The Author(s) 2020 